\title{
Near infra-red light treatment of Alzheimer's disease
}

\author{
Mengmeng Han*, Qiyan Wang*, Xue Wang*, Yuhui Zeng ${ }^{\dagger}$, Yong Huang ${ }^{\dagger}$, \\ Qingqiang Meng ${ }^{\dagger}$, Jun Zhang* and Xunbin Wei*, \\ ${ }^{*}$ Med-X Research Institute and School of Biomedical Engineering \\ Shanghai Jiao Tong University, 1954 Huashan Road \\ Shanghai 200030, P. R. China \\ $\nmid$ Zhejiang BrainHealth Medical Technology Co. \\ Ltd 332 Wangjiang Road, Hangzhou 310002, P. R. China \\ $\$$ Department of Neurosurgery \\ General Hospital of PLA 28 Fuxing Road \\ Beijing 100853, P. R. China \\ ${ }_{\text {¿xwei01@sjtu.edu.cn }}$
}

Received 30 July 2016

Accepted 22 February 2017

Published 14 April 2017

\begin{abstract}
Alzheimer's disease (AD) is a chronic neurodegenerative disease. The symptoms include memory and spatial learning difficulties, language disorders, and loss of motivation, which get worse over time, eventually ending in death. No effective treatments are available for $\mathrm{AD}$, currently. Current treatments only attenuate symptoms temporarily and are associated with severe side effects. Near infra-red (NIR) light has been studied for a long time. We investigated the effect of NIR on AD using a transgenic mouse model, which was obtained by co-injecting two vectors carrying $\mathrm{AD}$ mutations in amyloid precursor protein (APP) and presenilin-1 (PSEN1) into C57BL/6J mice. The irradiation equipment consisted of an accommodating box and an LED array. The wavelength of NIR light emitted from LED was between $1040 \mathrm{~nm}$ and $1090 \mathrm{~nm}$. The power density delivered at the level of the mice was approximately $15 \mathrm{~mW} / \mathrm{cm}^{2}$. Firstly, we treated the mice with NIR for 40 days. Then, the irradiation was suspended for 28 days. Finally, another 15 days treatment was brought to mice. We conducted Morris water maze and immunofluorescence analysis to evaluate the effects of treatment. Immunofluorescence analysis was based on measuring the quantity of plaques in mouse brain slices. Our results show that NIR light improves memory and spatial learning ability and reduces plaques moderately. NIR light represents a potential treatment for $\mathrm{AD}$.
\end{abstract}

Keywords: Alzheimer's disease; near infra-red light; transgenic mouse model; Morris water maze; immunofluorescence analysis.

$\S_{\text {Corresponding author. }}$

This is an Open Access article published by World Scientific Publishing Company. It is distributed under the terms of the Creative Commons Attribution 4.0 (CC-BY) License. Further distribution of this work is permitted, provided the original work is properly cited. 


\section{Introduction}

Alzheimer's disease (AD) tends to occur in people aged above 65 years. $^{1}$ It is a neurodegenerative disease and difficult to cure. Current therapeutic methods, mainly including medications, have limited effects in attenuating symptoms. No treatment can reverse the progression of disease. The pathogenesis of $\mathrm{AD}$ is still poorly understood. It is believed that amyloid- $\beta(\mathrm{A} \beta)$ deposition leads to the incidence and deterioration of $\mathrm{AD} .^{2} \mathrm{~A} \beta$ is derived from the regulated intracellular proteolysis of amyloid precursor protein (APP), which extensively exists in tissues. APP is hydrolyzed by $\alpha^{-}, \beta-$, and $\gamma$-secretase. Hydrolysis of APP by $\beta$-, and $\gamma$-secretase leads to $\mathrm{A} \beta$ formation. $\mathrm{A} \beta$ peptides contain 39-43 amino acids each, and $\mathrm{A} \beta_{1-40}$ and $\mathrm{A} \beta_{1-42}$ are the major peptides associated with $\mathrm{AD}$ plaque formation via aggregation. $\mathrm{A} \beta$ and senile plaques have long been considered neurotoxic and associated with the progression of $\mathrm{AD} .^{3}$ Plaque reduction is the therapeutic goal currently.

Light has been used for medical applications for a very long time. Near infra-red (NIR) light, a section of electromagnetic spectrum, has good performance in detection and treatment. The determination of NIR spectroscopy is useful for quantitative analysis of glycated hemoglobin and biomedical imaging in living tissues. ${ }^{4-7}$ NIR is therapeutic, due in part to its deep tissue penetration and low light scattering. NIR is typically applied with its thermal effects or biological effects in treatment. The heat converted from NIR helps the release of drug in microcapsule system. ${ }^{8}$ Biological effects of light include wound healing, pain reduction, and alleviation of oral mucositis. ${ }^{9-12}$ Effects such as cytoprotection, cellular proliferation, growth factor release have also been reported. ${ }^{13-15}$ Studies have indicated the effects of NIR on nervous system, like AD. ${ }^{16-19}$ The biological effects of NIR are specific to wavelength and not based on thermal effects. Those studies showed NIR mitigated AD neuropathology and behavioral deficits. For example, the CD1 mouse model treated with $1072 \mathrm{~nm}$ low infra-red light shows improvements in emotional responses and memory performance. ${ }^{16}$ AD mice which had undergone five month treatment with $1072 \mathrm{~nm}$ NIR showed a significant reduction of $\mathrm{A} \beta_{1-42}$ in the cerebral cortex and up-regulation of stress response proteins in the brain, which were known to reduce protein aggregation and neuronal apoptosis. ${ }^{17}$
The $808 \mathrm{~nm}$ wavelength significantly reduced amyloid plaques and improved behavioral performance in AD mouse model. ${ }^{18}$ Purushothuman et al. applied $670 \mathrm{~nm}$ NIR to two kinds of transgenic mouse models, finding out that NIR was associated with a reduction in hyperphosphorylated tau, neurofibrillary tangles, oxidative stress markers and $\mathrm{A} \beta$ plaques in the neocortex and hippocampus. ${ }^{19}$

We used a transgenic AD mouse model to evaluate the effect of NIR. The mouse model was coinjected with two mutant gene vectors involving APP and PSEN1. APP and PSEN1 mutations increase $\mathrm{A} \beta$ formation. ${ }^{20}$ Our transgenic model exhibits plaques and memory deficits at an early age. In this present study, we treated the AD mouse model using NIR light and analyzed the effects through Morris water maze (MWM) and immunofluorescence analysis (IFA). We chose different irradiation parameters from previous reports and designed a suspension between two periods of irradiation to assess the sustainability of the effect of NIR.

\section{Materials and Methods}

\subsection{Animals}

All our mice were obtained from the Institute of Laboratory Animal Science, Chinese Academy of Medical Sciences. The transgenic mouse model used in this study was produced by co-injecting the APPswe and PS1 $\triangle \mathrm{E} 9$ vectors. The APPswe vector expresses a mouse-human hybrid transgene containing the extracellular and intracellular regions of the mouse sequence as well as a human sequence within the $\mathrm{A} \beta$ domain with Swedish mutations (K594N/M595L). The PS1 $\Delta \mathrm{E} 9$ vector expresses the human presenilin-1 deleted exon-9. The background strain of the mice was C57BL/6J. ${ }^{21}$ The transgenic model showed memory and spatial learning disability as well as detectable plaques at an early age. Senile plaques were detected as early as 4.5 months. And behavioral differences between the model and wild-type mice appeared in three months. ${ }^{22}$ All the animals were female and divided into three different groups $a d(n=10), a d+i r(\mathrm{n}=$ $10)$ and $w t(n=12)$. Group $a d$ was transgenic mice and did not received irradiation. Group $a d+i r$ was transgenic mice and received irradiation. Group $w t$ was normal mice and did not received irradiation. All the mice were raised under $12 \mathrm{~h}$ light $/ 12 \mathrm{~h}$ dark cycle, and exposed to light from 7 am to $7 \mathrm{pm}$. 
The room temperature was maintained constant at $23 \pm 2{ }^{\circ} \mathrm{C}$. Mice were provided free access to water and food, and their cages were cleaned twice a week. Animal care and experimental protocols were in accordance with guidelines issued by the Shanghai Medical Experimental Animal Care Commission. At the start of the MWM task, we found four and two dead mice in the $a d+i r$ and $a d$ groups, respectively. Mice in the $a d+i r$ group died because of disrupted air supply, while mice in other groups were not affected. Two ad mice were attacked by others in the same cage. All the six mice died, but not because of IR irradiation. This study was approved by the Ethical Committee of Animal Experiments of Med-X Research Institutes, Shanghai Jiao Tong University.

\subsection{Methods and treatment}

Our patented apparatus (ZL 2015220692323.1 ) consists of a box containing mice and an LED array, which emits NIR light. The LED array also acts as the lid. The side windows and vertical openings provide air, food and water. We irradiated the $a d+$ ir group of mice six min per day for 40 days, at the age of six months, with a power density of approximatly $15 \mathrm{~mW} / \mathrm{cm}^{2}$ and a wavelength between $1040 \mathrm{~nm}$ and $1090 \mathrm{~nm}$. We chose $6 \mathrm{~min}$ according to previous studies. ${ }^{16,17}$ Then, we stopped the treatment and tested the memory and spatial learning ability of all the mice using a Morris water maze. The time mice spent was seven days in the first MWM test, among which the first two days were pre-experiment and helped mice to get familiar with the task. In order to study whether the irradiation effect will disappear, we suspended the irradiation for 28 days and conducted a second MWM task in all the three groups for five days. After that the $a d$ + ir mice were treated with 15 days of additional NIR light based on the assumption that 28 day suspension of irradiation would eliminate the effect of NIR treatment. Finally, we conducted the IFA to study the effect of irradiation on senile plaques in mouse brains. The study paradigm of the experiments is shown in Table 1.

\subsection{Morris water maze}

MWM is a widely used behavioral task in neuroscience to study memory and spatial learning ability of rodents. ${ }^{23}$ Morris described the basic procedures in 1984, followed by additional protocols for the assessment of related forms of learning and memory. ${ }^{24}$ The task is based on the latency time of mouse from the starting point to the platform. Increased latency suggests lower levels of memory and spatial learning ability. The MWM consists of a swimming pool (diameter, $120 \mathrm{~cm}$; height, $50 \mathrm{~cm}$ ) equipped with a submerged platform (diameter, $6 \mathrm{~cm}$ ) $1 \mathrm{~cm}$ below the surface of water. The circular pool is filled with water at $22 \pm 1^{\circ} \mathrm{C}$. The room temperature is maintained at a constant level. We used the classic protocol as described in a previous review. ${ }^{25} \mathrm{We}$ tested the mice for five days conducting four trials daily. In a single trial, the mouse was transferred to the pool from the start point and allowed $60 \mathrm{~s}$ to swim freely. The start points along the perimeter were arranged differently each day. The time taken by the mouse to locate the platform successfully within $60 \mathrm{~s}$ was recorded as the latency time. Otherwise, the latency time was counted as $60 \mathrm{~s}$. We consider the trial complete when the mouse located the platform or after a lapse of $60 \mathrm{~s}$. The mouse was relocated at the platform for $15 \mathrm{~s}$ to acclimatize with the surroundings. Each mouse was tested over four trials consecutively, daily. The software we used to record data was ANY-maze 4.99 (Stoelting Co.).

\subsection{Immunofluorescence analysis}

All the mice were euthanized via $\mathrm{CO}_{2}$ narcosis by rapidly dissecting the brain. The brains were

Table 1. The study paradigm of treatment and MWM experiments. The time arragement is shown from column 3 to column 7 and the unit was day.

\begin{tabular}{lcccccccc}
\hline Group & $\begin{array}{c}N \text { before } \\
\text { treatment }\end{array}$ & $\begin{array}{c}\text { First } \\
\text { irradiation }\end{array}$ & First MWM & $\begin{array}{c}\text { Irradiation } \\
\text { suspension }\end{array}$ & Second MWM & $\begin{array}{c}\text { Second } \\
\text { irradiation }\end{array}$ & $\begin{array}{c}N \text { after } \\
\text { treatment }\end{array}$ & $\begin{array}{c}\text { IFA } \\
\text { ad }+i r\end{array}$ \\
\hline ad & 10 & $1-40$ & $41-47$ & $41-68$ & $69-73$ & $74-88$ & 8 & Yes \\
wt & 10 & No & $41-47$ & No & $69-73$ & No & 6 & Yes \\
\hline
\end{tabular}


immediately frozen in iso-pentane and stored at $-80^{\circ} \mathrm{C}$. Brains were cut into coronal sections of $20-\mu \mathrm{m}$ thickness at $-20^{\circ} \mathrm{C}$. Endogenous peroxidase activity was inhibited by incubating the sections in $0.3 \%$ peroxide in methanol for $20 \mathrm{~min}$ at room temperature. The samples were subjected to three 5-min washes with phosphate buffer saline (PBS). The sections were fixed with $4 \%$ paraformaldehyde (PFA) in PBS (pH7.4) for 15 min and washed for $5 \mathrm{~min}$ three times, again. Subsequently, the sections were blocked with $10 \%$ normal donkey serum in $0.1 \%$ Triton X-100 for $30 \mathrm{~min}$ and incubated with the primary antibody (Abcam, ab2539, $1: 100)$ in $1 \%$ normal donkey serum overnight at $4{ }^{\circ} \mathrm{C}$. The primary antibody was sensitive to synthetic peptides corresponding to human beta amyloid aa 1-14 and reacted with all types of $\mathrm{A} \beta$. Before incubation with a second antibody, the samples were washed for $5 \mathrm{~min}$ three times. The anti-rabbit second antibody (Jackson, anti-rabbit $488 \mathrm{~nm}$, 1:400) was used in 1\% normal donkey serum for $1 \mathrm{~h}$ at room temperature followed by a 5-min wash three times. The slices were mounted using a mounting medium with DAPI (Vector laboratories, H-1200), commonly used for nuclear staining. Finally, the sections were imaged by the Leica fluorescence microscope at an excitation wavelength of about $360 \mathrm{~nm}$ to locate the nuclei in the hippocampus. Then, we switched the wavelength to $488 \mathrm{~nm}$ to count the plaques.

We analyzed the hippocampus and cerebral cortex of mouse brain for immunofluorescence because $\mathrm{A} \beta$ was mainly deposited in these areas. ${ }^{26}$ The plaques within the regions were divided into two categories depending on the counting sizes: small $(<20 \mu \mathrm{m})$ and large $(\geq 20 \mu \mathrm{m})$. Data were derived from three pictures from each mouse held in a fixed position of hippocampus to compare different mice. Plaques were counted manually and repeated three times. The counting was blinded to experimental paradigms. The number of plaques indicate the degree of $\mathrm{AD}$ severity. Therefore, we counted the number of plaques in the three groups to assess the effects of NIR irradiation therapy.

\subsection{Statistical analysis}

The data of each mouse in MWM were averaged and expressed as mean \pm SEM. We analyzed the data with SPSS to compare the means of different groups using two-way ANOVA, with group representing the between-subject factor, and time as the withinsubject factor. LSD post-hoc analysis was used to evaluate the differences between $a d+i r$ and the other two groups. In IFA, we analyzed the data through two-way ANOVA with Graphpad followed by Bonferroni post-hoc analysis.

\section{Results}

The results of the first round MWM are presented in Fig. 1. Figure 1(a) shows the column graph of the first round MWM data. Overall, the daily duration of the three groups appeared in the same sequence, from large to small. The ad group lasted longer than the $a d+i r$ group. And the latency time of $a d+i r$ group was longer than that in $w t$. Significant differences were found between $a d$ and $a d+i r$ groups on days 2 and 3 while significant differences between $a d+i r$ and $w t$ groups were observed on days 2,3 and 4 . The time that the mouse spent in the MWM

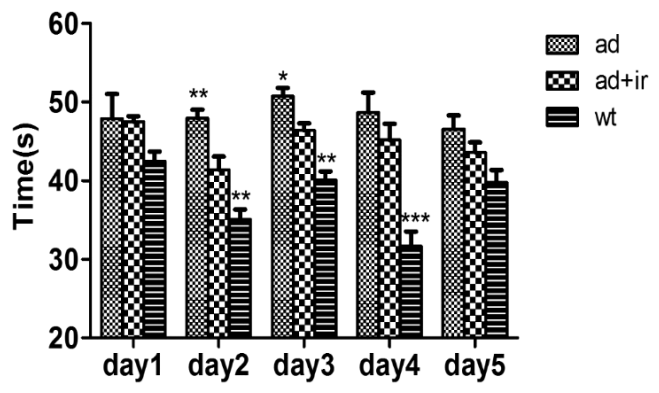

(a)

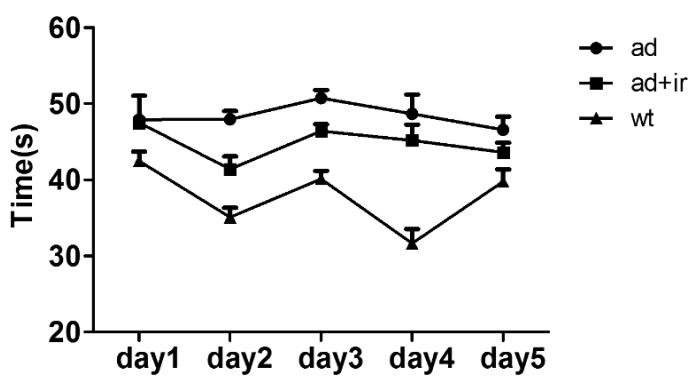

(b)

Fig. 1. The results of the first round MWM task. (a) The column graph of first round MWM data. (b) The line chart of first round Morris water data. All data are expressed as means \pm SEM. We compared the means of ad $(n=8)$ and $w t(n=12)$ with that of $a d+i r(n=6)$ using two-way ANOVA followed by LSD post-hoc $(* p<0.05, * * p<0.01$, $* * * p<0.001)$. 
task indicated the level of its memory and spatial learning ability. More capable the mouse was, the smaller the value of latency time was. Therefore, the constant sequence showed that the $a d+i r$ mice performed better than the $a d$ mice, which was worse than mice in $w t$ group. The average time of group $a d+i r$ was $44.8 \mathrm{~s}$, which was $7.4 \%$ less than the average time of group ad and $18.5 \%$ more than the average time of group $w t$. The two-way ANOVA showed $p$ value between $a d$ and $a d+i r$ of group factor was 0.0049. Figure 1(b) shows the line chart of the first round MWM. The line connects the means of daily data and reveals the variation trend in latency time. The height of the line represents the overall performance of the group. Two lines were separated from each other, clearly showing the differences in their means. All the results indicated that mice in the $a d+i r$ group performed better than those in ad after phototherapy, suggesting that the NIR light might be effective for $\mathrm{AD}$ treatment.

Figure 2 shows the results obtained from the second round of MWM after 28 days of treatment

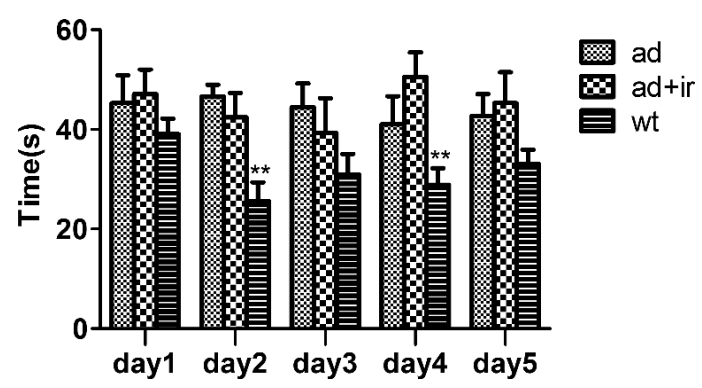

(a)

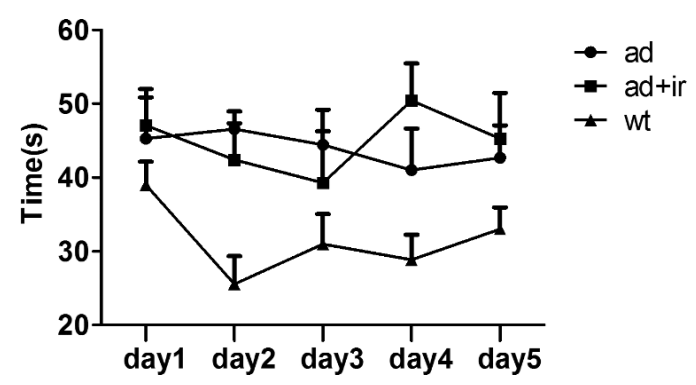

(b)

Fig. 2. The results of the second round MWM task. (a) The column graph of second round MWM data. (b) The line chart of second round Morris water data. All data represent means \pm SEM. We compared means of group $a d(n=8)$ and $w t(n=12)$ with that of group $a d+i r(n=6)$ using a two-way ANOVA followed by LSD post-hoc $\left({ }^{*} p<0.05,{ }^{* *} p<0.01,{ }^{* * *} p<0.001\right)$. suspension. As shown in Fig. 2(a), the column graph of the second round MWM revealed no significant difference between $a d$ and $a d+i r$ groups. Figure 2(b) presents the line chart of the second round MWM. The $a d$ and $a d+i r$ groups were almost similar and intertwined with each other. The second-round MWM data suggested that after 28 days of light therapy suspension, the $a d+i r$ group performance was almost same to that of $a d$, which was not irradiated. Therefore, these results indicated that the effects of IR treatment disappeared over time.

After the behavioral experiments, we used biochemical techniques to analyze the secretion of $\mathrm{A} \beta$ by labeling the plaques and counting their numbers in mouse brains. Immunofluorescence analysis was conducted according to standard procedures. The primary antibody used was sensitive to the synthetic peptide corresponding to human betaamyloid amino acids 1-14 conjugated to Keyhole Limpet Haemocyanin (KLH). An interesting study showed that both newly formed and existing plaques grew at a similar rate of $0.3 \mu \mathrm{m}$ (radius) per week. ${ }^{27}$ We assumed that IR treatment decreased amyloid plaques by interfering with the formation of new plaques. Therefore, we divided the plaques into two categories depending on their size: small $(<20 \mu \mathrm{m})$ and large $(\geq 20 \mu \mathrm{m})$.

The results of IFA are shown in Fig. 3. Figures 3(a) and $3(\mathrm{~b})$ illustrate immunofluorescence results. Figure 2(c) shows the column graph of ad $(n=4)$ and $a d+\operatorname{ir}(n=3)$ data. No plaques were found in $w t$ mouse brain. As shown in Fig. 3, mice in the $a d+$ ir group obviously showed fewer plaques than those in the ad group, with an average number of 15.33 and 35.5 in $a d+i r$ and $a d$ groups, respectively. Due to small sample size, the variance was not adequate to generate significant differences between the two groups. The mean number of plaques measuring $<20 \mu \mathrm{m}$ was 9 and 26 in $a d+i r$ and $a d$ groups, respectively. And the average number of plaques measuring $\geq 20 \mu \mathrm{m}$ was 11 and 6.3 in ad and $a d+i r$ groups, respectively. NIR treatment modestly reduced the small-size plaques, which was consistent with a previous study. ${ }^{19}$ There was significant difference $(p<0.05)$ between the two groups for plaques measuring $<20 \mu \mathrm{m}$. And for plaques measuring $\geq 20 \mu \mathrm{m}, p$ value was bigger than 0.05. The small sample size and short duration of irradiation might contribute to large $p$ value. More mice need to be tested in future studies. 


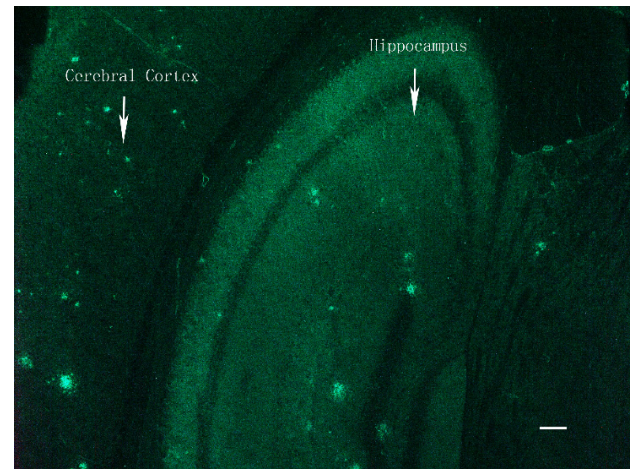

(a)

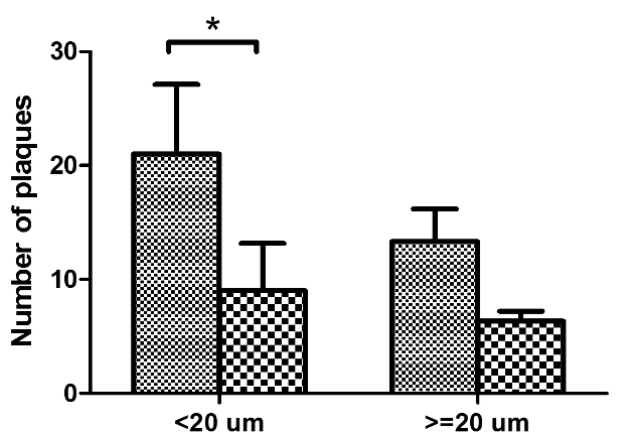

(c)

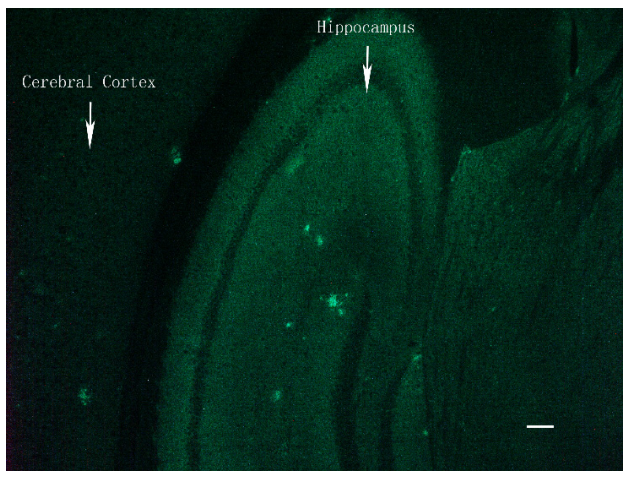

(b)

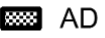

$\infty A D+I R$

Fig. 3. Immunofluorescence in hippocampus and cerebral cortex of mouse brains: $a d(n=4)$ and $a d+i r(n=3)$ groups. Scale bar $=100 \mu \mathrm{m}$. Representative images of (a) $a d$, and (b) $a d+i r$ groups of mice; (c) Statistical analysis of plaques in the two groups. Plaques were divided into two categories: $\geq 20 \mu \mathrm{m}$ and $<20 \mu \mathrm{m}$. All the data represent means \pm SEM. There was significant difference $(p<0.05)$ between the two groups for plaques measuring $<20 \mu \mathrm{m}$. And for those measuring $\geq 20 \mu \mathrm{m}, p$ value was bigger than 0.05 .

\section{Discussion and Conclusion}

Michalikova et al. administered $1072 \mathrm{~nm}$ infra-red light to CD1 mice to test their emotional response and memory performance. Ten days of exposure improved the working memory of mice. ${ }^{16}$ Another study reported by Grillo also employed $1072 \mathrm{~nm}$ to treat a transgenic mouse model over five months. ${ }^{17}$ In contrast to this chronic and low-power treatment protocol with $5 \mathrm{~mW} / \mathrm{cm}^{2}$, we adopt a daily therapy using a higher power density with about $15 \mathrm{~mW} / \mathrm{cm}^{2}$. Purushothuman et al. studied two mouse models with NIR treatment, and found out that NIR treatment could reduce hyperphosphorylated tau, neurofibrillary tangles, oxidative stress markers and plaques. ${ }^{19}$ They then extended the irradiated area to cerebellum. After one month of treatment, NIR mitigated AD-related pathologies. ${ }^{28}$ The mechanisms associated with NIR effect on $\mathrm{AD}$ mouse are typically mediated via ATP generation to increase and improve mitochondrial function. ${ }^{29}$ Taboada et al. used $808 \mathrm{~nm}$ in $\mathrm{A} \beta \mathrm{PP}$ transgenic mice, which increased the ATP levels as well as percent oxygen consumption. ${ }^{18}$ Previous reports suggest that early stage plaques could be more toxic than those at later stages. ${ }^{30}$ The early damage essentially involved cellular processes resulting in cell death. NIR treatment diminished cell death associated with amyloid plaque formation in transgenic mice, resulting in improved spatial learning and memory.

Though the NIR we used penetrated tissues deeply compared to other wavelengths, ${ }^{31}$ the unshaved fur of mice is still a major limitation because black fur blocks a major portion of the light. Considering the fur and skull of humans are thicker than those of mice, the power density could be higher. Shaving hair may help improve the penetration of NIR and the therapeutic effect. In addition, our conclusions were mainly based on $\mathrm{A} \beta$ deposits. Additional histological endpoints are needed considering the limitations associated with a 
single standard. Those histological endpoints include phosphorylated tau protein, heat-shock protein, inflammatory markers and so on. In our subsequent study, another group of $a d+i r$ which receive 40 day irradiation only could be included to check the development of plaques after the 40 day period and also to eliminate the confounding effects of the two separate NIR treatments.

Our study applied different irradiation parameters and designed a suspension between two periods of irradiation. Results showed that the parameters we chose worked well and the NIR effect was not permanent. Both provided important insights into clinical application of NIR on treating AD.

\section{Acknowledgments}

This work was supported by grants awarded by the National Major Scientific Research Program of China (Grant No. 2011CB910404), the National Nature Science Foundation of China (Grant No. 61227017), the State Key Basic Research Development Program of China (2012CB518103), and National Outstanding Young Scientist Award of China (61425006). We thank Professor Hao He, Dan Wei, Yuanzhen Suo, Zhangru Yang, Ping Yang, Zhenyu Niu, Xiaoling Wang, Liuying Chai, Pan Cheng, Xi Zhu for helpful discussions. We also thank Professor Guoyuan Yang and his students for providing the Morris water maze.

\section{References}

1. A. Burns, S. Iliffe, "Alzheimer's disease", BMJ Clin. Res. ed. 338, b158 (2009).

2. J. Hardy, D. Allsop, "Amyloid deposition as the central event in the aetiology of Alzheimer's disease," Trends Pharmacol. Sci. 12, 383-388 (1991).

3. Karen H., P. Chapman, S. Nilsen, C. Eckman, Y. Harigaya, S. Younkin, F. S. Yang, G. Cole, "Correlative memory deficits, $\mathrm{A} \beta$ elevation, and amyloid plaques in transgenic Mice," Science $\mathbf{2 7 4}$ (5284), 99-103 (1996).

4. T. Pan, M. M. Li, J. M. Chen, H. Y. Xue, "Quantification of glycated hemoglobin indicator HbA1c through near-infrared spectroscopy," $J$. Innov. Opt. Health Sci. 7(4), 1350060 (2014).

5. X. Zhang, Y. Q. Gu, H. Y. Chen, "Synthesis of biocompatible near infrared fluorescence $\mathrm{Ag}_{2} \mathrm{~S}$ quantum dot and its application in bioimaging," $J$. Innov. Opt. Health Sci. 7(3), 1350059 (2014).
6. K. A. Kang, "Britton Chance's lab and thereafter: From NIR spectroscopy to molecular sensing via nanotechnology", J. Innov. Opt. Health Sci. 7(2), 1330004 (2014).

7. M. A. Yucel, J. Selb, R. J. Cooper, D. A. Boas, "Targeted principle component analysis: A new motion artifact correction approach for near-infrared spectroscopy," J. Innov. Opt. Health Sci. 7(2), 1350066 (2014).

8. Y. F. Di, S. S. Cui., Y. Q. Gu, "Preparation and characterization of a near-infrared light responsive microcapsule system for cancer therapy," J. Innov. Opt. Health Sci. 7(1), 1350037 (2014).

9. J. T. Eells, M. T. Wong-Riley, J. VerHoeve, M. Henry, E. V. Buchman, M. P. Kane, L. J. Gould, R. Das, M. Jett, B. D. Hodgson, D. Margolis, H. T. Whelan, "Mitochondrial signal transduction in accelerated wound and retinal healing by near-infrared light therapy," Mitochondrion 4(5-6), 559-567 (2004).

10. E. Mester, T. Spiry, B. Szende, J. G. Tota, "Effect of laser rays on wound healing," Am J. Surg. 122(4), 532-535 (1971).

11. A. Honmura, A. Ishii, M. Yanase, J. Obata, E. Haruki, "Analgesic effect of Ga-Al-As diode laser irradiation on hyperalgesia in carrageenan-induced inflammation," Lasers Surg. Med. 13(4), 463-469 (1993).

12. H. T. Whelan, J. F. Connelly, B. D. Hodgson, L. Barbeau, A. C. Post, G. Bullard, E. V. Buchman, M. Kane, N. T. Whelan, A. Warwick, D. Margolis, "NASA light-emitting diodes for the prevention of oral mucositis in pediatric bone marrow transplant patients," J. Clin. Laser Med. Surg. 20(6), 319-324 (2002).

13. A. Bradford, A. Barlow, L. P. Chazot, "Probing the differential effects of infrared light sources IR 1072 and IR 880 on human lymphocytes: Evidence of selective cytoprotection by IR 1072," J. Photochem. Photobiol. B, Biol. 81, 9-14 (2004).

14. M. J. Conlan, J. W. Rapley, C. M. Cobb, "Biostimulation of wound healing by low-energy laser irradiation," A Rev. J. Clin. Periodontol. 23, 492-496 (1996).

15. M. C. P. Leung, S. C. L. Lo, F. K. W. Siu, K. F. So, "Treatment of experimentally induced transit cerebral ischemia with low energy laser inhibits nitric oxide synthase activity and up-regulates the expression of transforming growth factor-beta1," Lasers Surg. Med. 31, 283-288 (2002).

16. S. Michalikova, A. Ennaceur, R. van Rensburg, P. L. Chazot, "Emotional responses and memory performance of middle-aged CD1 mice in a 3D maze: Effects of low infrared light," Neurobiol. Learn. Mem. 89, 480-488 (2008). 
17. S. L. Grillo, N. A. Duggett, A. Ennaceur, P. L. Chazot, "Non-invasive infra-red therapy (1072 nm) reduces $\beta$-amyloid proteins levels in the brain of an Alzheimer's disease mouse model, TASTPM," J. Photochem. Photobiol. B, Biol. 123, 13-22 (2013).

18. D. T. Luis, Y. Jin, E. Salim, G. Sebastiano, R. Steve, M. Thomas, S. Jackson, K. Mark, "Transcranial laser therapy attenuates amyloid- $\beta$ peptide neuropathology in amyloid- $\beta$ protein precursor transgenic mice," J. Alzheimer's Dis. 23, 521-535 (2011).

19. S. Purushothuman, D. M. Johnstone, C. Nandasena, J. Mitrofanis, J. Stone, "Photobiomodulation with near infrared light mitigates Alzheimer's diseaserelated pathology in cerebral cortex - evidence from two transgenic mouse models," Alzheimer's Res. Ther. 6, 2 (2014).

20. D. J. Selkoe, "Translating cell biology into therapeutic advances in Alzheimer's disease," Nature 399, A23A31 (1999).

21. H. Wang, J. Liu, Y. Zong, Y. Xu, W. Deng, H. Zhu, Y. Liu, C. Ma, L. Zhang, C. Qin, "miR-106b aberrantly expressed in a double transgenic mouse model for Alzheimer's disease targets TGF- $\beta$ type II receptor," Brain Res. 1357, 166-174 (2010).

22. Y. Y. Zong, X. Y. Wang, H. L. Wang, Y. L. Liu, L. Huang, C. M. Ma, L. F. Zhang, C. Qin, "Continuous analysis of senile plaque and behaviour in APPswe/ PS $\Delta$ E9 double-transgenic gene mouse model of alzheimer's disease," Chin. J. Com. Med. 18(9), 8-12 (2008).

23. R. D' Hooge, P. P. De Deyn, "Applications of the Morris water maze in the study of learning and memory," Brain Research Rev. 36(1), 60-90 (2001).

24. R. Morris, "Developments of a water-maze procedure for studying spatial learning in the rat," $J$. Neurosci. Methods 11, 47-60 (1984).
25. C. V. Vorhees, M. T. Williams, "Morris water maze: Procedures for assessing spatial and related forums of learning and memory," Nat. Protoc. 1(2), 848858 (2006).

26. D. R. Howlett, K. Bowler, P. E. Soden, D. Riddell, J. B. Davis, J. C. Richardson, S. A. Burbidge, M. I. Gonzalez, E. A. Irving, A. Lawman, G. Miglio, E. L. Dawson, I. Hussain, "Abeta deposition and related pathology in an APP/PS1 transgenic mouse model of Alzheimer's disease," Histol. Histopathol. 23, 67-76 (2008).

27. J. K. Hefendehl, B. M. Wegenast-Braun, C. Liebig, D. Eicke, D. Milford, M. E. Calhoun, S. Kohsaka, M. Eichner, M. Jucker, "Longterm in vivo imaging of beta-amyloid plaque appearance and growth in a mouse model of cerebral beta-amyloidosis," $J$. Neurosci. 31, 624-629 (2011).

28. S. Purushothuman, D. M. Johnstone, C. Nandasena, J. van Eersel, L. M. Ittner, J. Mitrofanis, J. Stone, "Near infrared light mitigates cerebellar pathology in transgenic mouse models of dementia," Neurosci. Lett. 591, 155-159 (2015).

29. X. J. Wu, A. E. Dmitriev, M. J. Cardoso, A. G. Viers-Costello, R. C. Borke, J. Streeter, J. J. Anders, "810 nm wavelength light: An effective therapy for transected or contused rat spinal cord," Lasers Surg. Med. 41, 36-41 (2009).

30. M. Stefani, C. M. Dobson, "Protein aggregation and aggregate toxicity: New insights into protein folding, misfolding diseases and biological evolution," J. Mol. Med. 81, 678-699 (2003).

31. A. N. Yaroslavsky, P. C. Schulze, I. V. Yaroslavsky, R. Schober, F. Ulrich, H. J. Schwarzmaier, "Optical properties of selected native and coagulated human brain tissues in vitro in the visible and near infrared spectral range," Phys. Med. Biol. 47(12), 2059-2073 (2002). 